\title{
Smoking As a Vital Sign: Prompts to Ask and Assess Increase Cessation Counseling
}

\author{
Anna McCullough, MSW, MSPH, Michael Fisher, MD, MPH, \\ Adam O. Goldstein, MD, MPH, Kathryn D. Kramer, PhD, and \\ Carol Ripley-Moffitt, MDiv, CCPHC
}

Background: Strategies to improve smoking cessation counseling in clinical settings are critical to supporting smokers' attempts to quit. This study evaluates the impact of adding 2 smoking-related vital sign questions in an electronic medical records system on identification, assessment, and counseling for patients who smoke: "Current smoker?" and "Plan to quit?"

Methods: Baseline data and data after intervention were collected through record review of 899 randomly selected patient visits across 3 outpatient clinics.

Results: From before to after intervention, identification of smokers increased $18 \%$ (from $71 \%$ to $84 \% ; P<.001$ ), and assessment for a plan to quit increased $100 \%$ (from $25.5 \%$ to $51 \% ; P<.005$ ). Among all smokers, cessation counseling increased $26 \%$ (from $23.6 \%$ to $29.8 \% ; P=.41$ ). Significantly more smokers who received the assessment for a plan to quit received cessation counseling (46\% vs. $14 \%, P<.001)$. Regression analysis showed that patients receiving an assessment for plan to quit were $80 \%$ more likely to receive cessation counseling (OR 0.209; 95\% CI, 0.095- 0.456).

Conclusions: Physician-documented counseling rates are significantly higher when patients are asked about smoking and assessed for a plan to quit. Two questions that ask about smoking status and assess plans to quit may provide prompts to increase the likelihood that patients who smoke receive cessation counseling. (J Am Board Fam Med 2009;22:625-632.)

Physicians have a unique and powerful opportunity to improve the health of their patients by asking about smoking behavior and providing cessation advice and counseling to smokers at every visit. Seven out of 10 smokers visit a physician yearly, providing ample opportunity for physician-directed cessation counseling, which has been shown to increase quit rates. ${ }^{1-4}$ Between $37 \%$ and $70 \%$ of smokers report recalling a physician advising them to quit, ${ }^{1,5-6}$ but evidence suggests that far fewer smokers receive assistance with quitting in the form of cessation counseling from their health care provider. ${ }^{7,8}$ A recent analysis of data from the national

This article was externally peer reviewed.

Submitted 7 October 2008; revised 16 April 2009; accepted 21 April 2009.

From the Nicotine Dependence Program, Department of Family Medicine, University of North Carolina School of Medicine, Chapel Hill.

Funding: Research costs were funded by University of North Carolina Health Care, The University of North Carolina School of Medicine.

Conflict of interest: none declared.

Corresponding author: Anna McCullough, Department of Family Medicine, University of North Carolina at Chapel Hill, School of Medicine, Chapel Hill, NC 27599-7595 (E-mail: annamc@unc.edu).
Ambulatory Medical Care Survey found that, although rates of identification of smokers increased slightly between 1994 and 2003, rates of cessation counseling actually decreased slightly, to $20 \%$ of identified smokers. ${ }^{8}$

Newly updated clinical practice guidelines from the Department of Health and Human Services (DHHS) recommend that "clinicians and health care systems seize the office visit for universal assessment and intervention. Specifically, ask every patient who presents to a health care facility if $s / h e$ uses tobacco (Ask), advise all tobacco users to quit (Advise), and assess the willingness of all tobacco users to make a quit attempt at this time (Assess)." The guidelines recommend system-level interventions to facilitate universal identification, assessment, and treatment of tobacco users.

In 1991, Fiore $^{10}$ proposed a system intervention-the addition of smoking as a vital sign (SVS) - to improve rates of tobacco use identification and prompt increased cessation advice from providers. Although the use of an SVS question that asks about a patient's current smoking status has increased rates of tobacco use identification in many health care settings, ${ }^{11-14}$ the evidence that 
SVS increases the provision of cessation advice and counseling is mixed. Studies using patient report and record review reported no change in provider advice after the introduction of SVS, even with additional staff training. ${ }^{11-12}$ Other studies report increases in the provision of simple advice to quit after the introduction of a single SVS, but no improvements in more specific cessation counseling. ${ }^{13-15}$

Clinical practice guidelines recommend SVS as a systems strategy to achieve identification of all smokers during every health care encounter, ${ }^{9}$ but the mixed evidence cited above suggests that new approaches are needed to improve the limited effects of SVS on the provision of cessation counseling. Although clinical practice guidelines also recommend that every smoker be asked about their readiness or plan to quit, an assessment that should provide information to guide further cessation advice and counseling, little research has examined the impact of such assessment on counseling. One model for studying this question is to add a second SVS question about assessment or planning to quit, providing a prompt for health care providers to engage in providing more thorough advice of their patients who smoke.

This study is the first to evaluate the impact of adding 2 SVS questions to the electronic medical records (EMRs) in a large academic medical center. In addition to asking the traditional SVS question about smoking, a second question ("Plan to quit?") extends the possibility that a systematic intervention with an expanded SVS designed to assess smokers' readiness to quit may increase smoking cessation counseling. Outcome measures in this study included rates of smoking status identification and cessation counseling before and after introduction of the 2 SVS questions. This study offers an initial exploration of the relationship between assessment of smokers' readiness to quit and the provision of cessation counseling.

\section{Methods}

\section{Study Design and Overview}

Using a before and after intervention design, we evaluated the impact of adding 2 SVS questions to the Web Clinical Information System EMR at a large academic medical center. The 2 SVS questions-“Current smoker?" and "Plan to quit?"were added to the end of the traditional vital signs section in March 2007. Before the introduction of the SVS, no standardized structure for recording smoking status existed across clinics within the medical center.

Baseline data and data after intervention were gathered via EMR reviews of selected patient visits during 3 periods: November 2006, April 2007, and November 2007. We reviewed EMR visit notes from 1- to 2-week periods during each study period in the family medicine, general internal medicine, and gynecology outpatient clinics. In each clinic, a medical assistant or nurse was responsible for asking the SVS questions and recording them in the EMR; physicians received SVS information via a hard copy report attached to the encounter form.

\section{Sample}

For each study period, using the Microsoft Excel random number generator (Microsoft Corp., Redmond, WA), we selected patient encounters from all clinic visits. We excluded selected encounters from the study if patients were younger than 18 or older than 89 at the time of the study visit, if a physician's signed progress note was not available for the study visit date, or if patients had already had an encounter included in the study during that study period. After exclusions, the gynecology clinic had only 99 eligible patient encounters in November 2006; all other clinics had 100 eligible encounters, the target review number, for each study period. Across the 3 study periods, a total of 899 chart reviews occurred.

Data collected from chart reviews included demographic data (age, sex, ethnicity); insurance type (Medicaid, Medicare, third party, or none/selfpay); type of visit (preventive/well or problemfocused); and evidence that the patient had certain smoking-related illnesses. Chart reviews included patients seen by both resident and attending physicians.

Researchers classified patients as current smokers or nonsmokers by reviewing the study visit progress note, nursing note, the EMR active problem list, and the SVS (after intervention). If researchers identified the patient as a current smoker, they recorded where the documentation of smoking occurred (ie, progress note, nursing note, problem list, or SVS). Assessment of readiness to quit smoking was indicated by documentation in the second SVS question ("Plan to quit?") or by documentation in the progress note that the physician asked the patient if they wanted to quit or had a 


\begin{tabular}{|c|c|c|c|c|}
\hline Characteristics & $\begin{array}{l}\text { November } 2006 \\
\quad(\mathrm{n}=299)\end{array}$ & $\begin{array}{l}\text { April } 2007 \\
(\mathrm{n}=300)\end{array}$ & $\begin{array}{l}\text { November } 2007 \\
\quad(\mathrm{n}=300)\end{array}$ & $\begin{array}{c}\text { All } \\
(\mathrm{n}=899)\end{array}$ \\
\hline \multicolumn{5}{|l|}{ Sex } \\
\hline Female & 76.3 & 74.7 & 70.0 & 73.6 \\
\hline \multicolumn{5}{|l|}{ Age (years)* } \\
\hline $18-35$ & 26.4 & 25.3 & 16.7 & 22.8 \\
\hline $36-64$ & 55.5 & 56.3 & 65.3 & 59.1 \\
\hline $65-89$ & 18.1 & 18.3 & 18.0 & 18.1 \\
\hline \multicolumn{5}{|l|}{ Race } \\
\hline Black & 29.4 & 34.0 & 30.3 & 31.3 \\
\hline White & 60.2 & 54.7 & 58.3 & 57.7 \\
\hline Other $^{\dagger}$ & 10.4 & 11.3 & 11.3 & 11.0 \\
\hline \multicolumn{5}{|l|}{ Type of insurance } \\
\hline Medicaid & 8.0 & 7.1 & 8.7 & 7.9 \\
\hline Medicare & 24.4 & 28.3 & 26.0 & 26.2 \\
\hline Private/third party & 42.5 & 36.4 & 40.7 & 39.8 \\
\hline None/self-pay & 25.1 & 28.3 & 24.7 & 26.0 \\
\hline \multicolumn{5}{|l|}{ Type of visit } \\
\hline Preventive/well & 12.7 & 8.3 & 14.7 & 11.9 \\
\hline Problem-focused & 87.3 & 91.7 & 85.3 & 88.1 \\
\hline \multicolumn{5}{|l|}{ Type of clinician ${ }^{\ddagger}$} \\
\hline Attending & 49.5 & 53.0 & 73.0 & 58.5 \\
\hline Resident & 50.5 & 47.0 & 27.0 & 41.5 \\
\hline \multicolumn{5}{|c|}{ Presence of chronic illness } \\
\hline No illness & 49.2 & 43.3 & 40.7 & 44.4 \\
\hline At least one illness & 50.8 & 56.7 & 59.3 & 55.6 \\
\hline
\end{tabular}

All data provided as \%.

${ }^{*}$ Differences between 3 data collection periods significant at $P<.05$.

+"Other" includes all patients whose race was recorded on EMR as American Indian, Asian, Hispanic, or Other.

${ }^{\ddagger}$ Differences between 3 data collection periods significant at $P<.001$.

plan to quit. Provision of cessation counseling was indicated by documentation in the progress note of at least one of the following: advice to quit, goal for quit date, provision of cessation educational materials, referral for cessation support, prescription of smoking cessation medication, or arrangement for smoking-specific follow-up. We selected these indicators based on current clinical guidelines and evidence-based interventions, such as engaging in problem-solving and coping skills or identifying sources of support within or outside of the patient/ provider relationship. ${ }^{16}$

\section{Data Analysis}

We combined data from the 2 periods after intervention to increase the sample size of smokers available. Data analysis comparing data before and after intervention data were performed using $\chi^{2}$ statistics to examine differences between groups (smokers versus nonsmokers and study period samples). We used $\chi^{2}$ analyses to assess differences in identification of smoking, assessment for plan to quit, and documentation of counseling before and after intervention and in relation to patient responses to the second SVS question ("Plan to quit?"). Binary logistic regression analysis examined associations between the independent variable "Plan to quit?" and the dependent variable "documented counseling" while adjusting for other independent variables. All analyses used 2-tailed $P=.05$ for significance and were performed using SPSS version 15.0 software (SPSS, Inc., Chicago, IL). The research was approved by the University of North Carolina School of Medicine Institutional Review Board.

\section{Results}

The demographic characteristics of the sample are shown in Table 1 . The majority of the sample was 


\begin{tabular}{|c|c|c|c|}
\hline Characteristics & $\begin{array}{l}\text { Current Smokers } \\
\quad(\mathrm{n}=159)\end{array}$ & $\begin{array}{l}\text { Nonsmokers } \\
(\mathrm{n}=556)\end{array}$ & $\begin{array}{l}\text { Smoking Data Missing } \\
(\mathrm{n}=184)\end{array}$ \\
\hline \multicolumn{4}{|l|}{$\operatorname{Sex}^{*}$} \\
\hline Female & 71.1 & 71.0 & 83.7 \\
\hline \multicolumn{4}{|l|}{ Age (years) ${ }^{\dagger}$} \\
\hline $18-35$ & 14.5 & 22.1 & 32.1 \\
\hline $36-64$ & 75.5 & 55.6 & 55.4 \\
\hline $65-89$ & 10.0 & 22.3 & 12.5 \\
\hline \multicolumn{4}{|l|}{ Race/ethnicity* } \\
\hline Black & 37.1 & 29.5 & 31.5 \\
\hline White & 59.7 & 58.6 & 53.3 \\
\hline Other & 3.2 & 11.9 & 15.2 \\
\hline \multicolumn{4}{|l|}{ Type of insurance $^{\dagger}$} \\
\hline Medicaid & 13.2 & 6.0 & 9.2 \\
\hline Medicare & 26.4 & 28.0 & 20.8 \\
\hline Private/third party & 20.1 & 44.8 & 42.1 \\
\hline None/self-pay & 40.3 & 21.2 & 27.9 \\
\hline \multicolumn{4}{|l|}{ Type of visit ${ }^{\dagger}$} \\
\hline Preventive/well & 4.4 & 15.5 & 7.6 \\
\hline Problem-focused & 95.6 & 84.5 & 92.4 \\
\hline \multicolumn{4}{|l|}{ Type of clinician ${ }^{\dagger}$} \\
\hline Attending & 47.2 & 63.3 & 53.8 \\
\hline Resident & 52.8 & 36.7 & 46.2 \\
\hline \multicolumn{4}{|c|}{ Presence of chronic illness ${ }^{\dagger}$} \\
\hline No illness & 31.4 & 44.2 & 56.0 \\
\hline At least one illness & 68.6 & 55.8 & 44.0 \\
\hline
\end{tabular}

All data provided as \%.

*Differences between groups significant at $P<.05$.

${ }^{\dagger}$ Differences between groups significant at $P<.001$.

‡"Other” includes all patients whose race was recorded on electronic medical record as American Indian, Asian, Hispanic, or other.

female, aged 36 to 54, white, covered by private or third-party insurance, and seen for a problemfocused visit. The second sample after intervention had significantly lower percentages of patients aged 18 to 35 and patients who saw resident physicians.

\section{Smoking Status}

A total of 159 current smokers were identified in the EMR from the study visit (Table 2). Six patients had documentation of spit tobacco use; for the purposes of this study spit tobacco users were classified as nonsmokers unless they also used cigarettes or other smoking materials. Information regarding current smoking status was missing from the study-visit EMR for 184 patients (20.5\%) out of the total sample; these patients were assumed to be nonsmokers for the purposes of this study. Of those patients whose smoking status was identified $(\mathrm{n}=$ 715 ), the overall rate of current smoking was $22.2 \%$ across the 3 study clinics. Current smokers ranged from $21.6 \%$ in the family medicine clinic to $23 \%$ in the internal medicine clinic. Current smoking rates were significantly lower in the second data collection period after intervention $(17.2 \%)$ than in the collection period before intervention $(25.9 \% ; P<$ $.05)$ and the first data collection period after intervention $(24.3 \% ; P=.05)$.

\section{Documentation of Smoking Status at Study Visit}

From periods before and after intervention, documentation of smoking status at the study visit increased $18 \%$ across all clinics (from $71 \%$ before intervention to $84 \%$ after intervention across all clinics; $P<.001)$ (Figure 1).

\section{Assessment of Plan to Quit}

For patients identified as current smokers, the assessment of a plan for cessation increased 100\% 


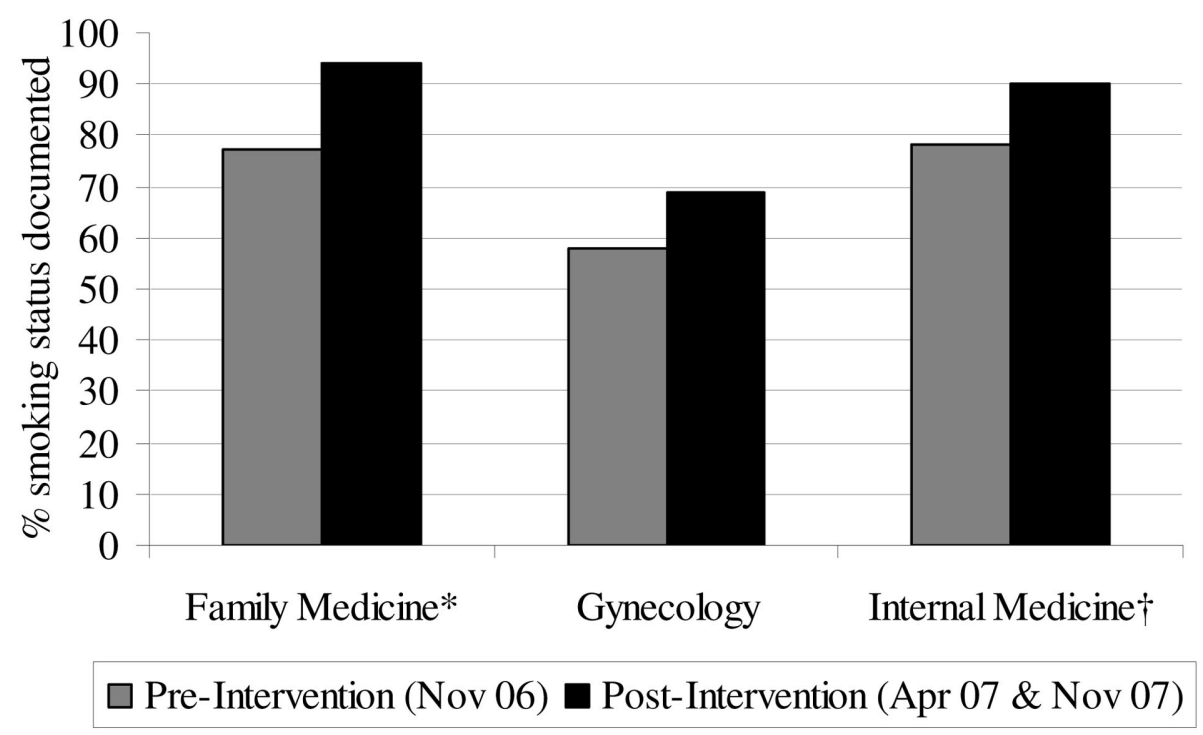

Figure 1. Proportion of medical records with tobacco status documented. *Differences between periods before and after intervention significant at $P<.001$. $†$ Differences between periods before and after intervention significant at $P<.05$.

(from $25.5 \%$ of current smokers before intervention to $51 \%$ of current smokers after intervention; $P<.005)$. After introduction of the SVS questions, 6 times more patients whose smoking status was documented in the SVS received an assessment for a plan to quit $(87 \% ; n=46)$ than patients whose smoking status was documented elsewhere in the EMR but not in the SVS $(13 \% ; \mathrm{n}=7 ; P<.001)$.

\section{Assistance with Cessation}

Documented counseling rates for all smokers increased 26\% after the SVS intervention (from $23.6 \%$ to $29.8 \%$; SVS $P=.41$ ). Figure 2 shows that, among the subset of smokers who received an assessment for having a plan to quit, nearly half $(46 \%)$ also received some form of cessation counseling compared with only $14 \%$ of smokers who did

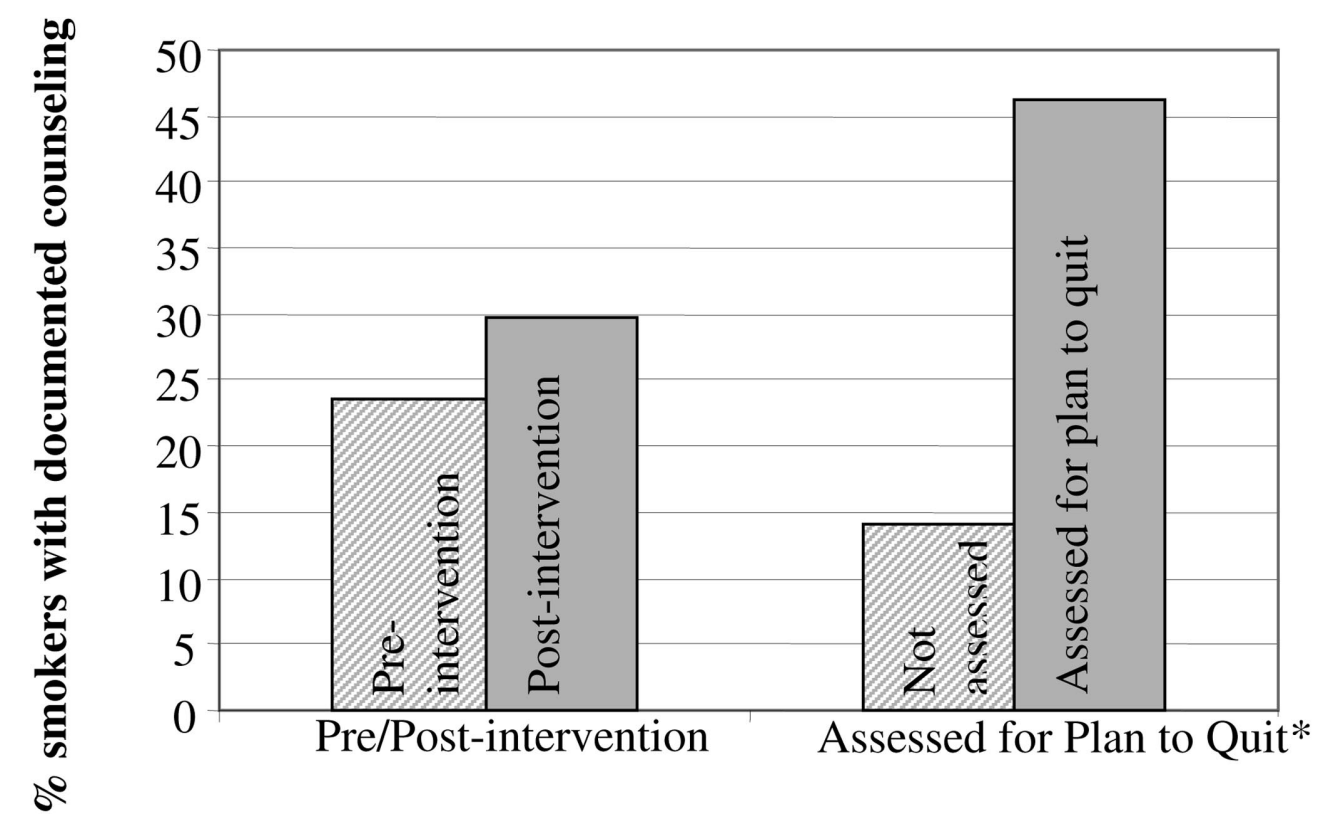

Figure 2. Documented counseling by time period and assessment for plan to quit. *Differences between groups significant at $P<.001$. 
not receive an assessment for having a plan to quit $(P<.001)$.

\section{Association between Plan to Quit Assessment and Documented Counseling}

Dependent variables were entered simultaneously into a binary logistic regression model as potential predictors of documented counseling across all time periods. They included age, sex, race, presence of a smoking-related chronic illness, type of clinician (attending or resident), type of visit (preventive or problem focused), and assessment for plan to quit.

Assessment for having a plan to quit was the only variable significantly associated with receiving cessation counseling (odds ratio, 0.209; 95\% CI, 0.095-0.456). Smokers who received assessment for having a plan to quit were $80 \%$ more likely than smokers who were not assessed to receive cessation counseling.

\section{Discussion}

Results from our study showed that the introduction of SVS to the EMR, using 2 questions to identify smokers and assess for a plan to quit, significantly increased identification of patient smoking. Although counseling rates did not increase significantly in the group overall (sample size calculation predicted a $70 \%$ increase in counseling and only a $28 \%$ increase was seen), counseling rates were significantly higher for smokers who received the assessment for a plan to quit in addition to being asked about their smoking behavior. This is one of the first studies to systematically examine 2 components of the recommended "5 As" incorporated as a vital sign through the EMR. Our findings suggest that a combination of vital sign questions to assess patients' smoking status and whether they currently have a plan for cessation are ultimately likely to be more effective prompts for provider cessation counseling than the traditionally used single-question SVS. The overall low rates of counseling observed in our study, $23 \%$ to $30 \%$, although comparable to those shown in the SVS literature, ${ }^{8,12,14-15}$ reinforce the need to increase utilization of systems interventions to increase use of these SVS questions across all relevant patients, in combination with other efforts to increase counseling. Systems interventions recommended in the updated DHHS clinical guidelines include provid- ing ongoing education and feedback about cessation counseling performance and designating staff to act as tobacco-dependence treatment coordinators. ${ }^{9}$

Several limitations to this study exist. One limitation is that other factors in addition to the introduction of SVS may have contributed to increased rates of smoking identification and cessation counseling during the study period. The hospital campus of the academic medical center went smokefree in July 2007, and 2 of the study clinics hosted Grand Rounds lectures about smoking cessation between the 2 follow-up study periods, including information about the addition of the SVS questions to the EMR. However, research on continuing medical education in didactic form has not shown a significant effect on provider behaviors or practice. ${ }^{17,18}$ Further, the increases seen in our study occurred almost exclusively among those smokers who were assessed for having a plan to quit. Varenicline was also introduced in July of 2006, and information about the drug was included in presentations about the tobacco-free policy, perhaps influencing the rate of cessation counseling. Given that the rate of prescription of smoking cessation medication was low in our study (5\% across all study clinics), it seems unlikely that the introduction of a new medication had a significant influence on counseling rates. Rates of counseling did not vary significantly between the 2 follow-up time periods, when most of the additional system and departmental efforts occurred.

Another limitation involves the use of medical record reviews to measure counseling rates. Studies have found that smoking cessation advice is documented in the medical record (both paper based and electronic) at significantly lower rates than it is reported by patients or directly observed. ${ }^{19-22}$ As such, physicians in this study may have failed to document cessation counseling, resulting in a lower rate of increase in documentation that may not accurately reflect real changes in the rate of counseling over time.

Finally, it is uncertain whether our findingsthat the combination of asking about smoking and assessing for a plan to quit, when performed and documented as prompted by 2 SVS questions, was associated with significantly higher counseling rates in our ambulatory clinics - can be generalized to other settings. The addition of single SVS questions in an urban hospital walk-in clinic and a 
research network of ambulatory care clinics resulted in significant improvements in the provision of simple cessation advice. ${ }^{13,14}$ However, singlequestion SVS interventions in other types of clinical settings had a negligible impact on counseling rates. To our knowledge, no studies examining the effect of 2 SVS signs have been published. Further research is needed to investigate the strength of the association reported here and to determine whether a 2-question or even a 3-question SVS intervention would be effective in other clinical settings. It is interesting to note that we heard of no negative feedback from nurses or physicians about the intervention.

\section{Conclusion}

Although overall documented counseling rates did not improve as much as predicted after the introduction of a 2-question SVS, the results show that when patients are both asked about smoking and assessed for readiness to quit, there is likely to be a significant increase in the incidence of physiciandocumented cessation counseling. Given that the addition of 2 SVS signs into our health care system's EMR occurred without system-wide training or other supports, the increases we observed when both the ask and assess steps were documented suggest that a more comprehensive approach to a double SVS system would have a more dramatic impact on the provision of cessation counseling. The updated DHHS clinical practice guidelines recommend that every patient be asked about tobacco use and assessed for willingness to quit as part of the recommended brief clinical intervention for smokers. ${ }^{9}$ Additional research is warranted for interventions that achieve both these steps as part of the vital signs in a medical record system, providing prompts that may increase the likelihood that physicians will provide substantively more cessation counseling.

The authors thank Tiffany Fisher and Stephen Daugird for their assistance with data collection.

\section{References}

1. Centers for Disease Control and Prevention. Physician and other health care professional counseling of smokers to quit-United States, 1991. MMWR Morb Mortal Wkly Rep 1993;42:854-7.

2. Gorin SS, Heck JE. Meta-analysis of the efficacy of tobacco counseling by health care providers. Cancer Epidemiol Biomarkers Prev 2004;13:2012-22.

3. Bao Y, Duan N, Fox SA. Is some provider advice on smoking cessation better than no advice? An instrumental variable analysis of the 2001 National Health Interview Survey. Health Serv Res 2006;41(6):21142135.

4. Stead LF, Bergson G, Lancaster T. Physician advice for smoking cessation. Chochrane Database Syst Rev 2008;(4):CD000165.

5. Anda RF, Remington PL, Sienko DG, Davis RM. Are physicians advising smokers to quit? The patient's perspective. JAMA 1987;257:1916-9.

6. Denny CH, Serdula MK, Holtzman D, Nelson D. Physician advice about smoking and drinking: are U.S. adults being informed? Am J Prev Med 2003; 24:71-4.

7. Ferketich AK, Khan Y, Wewers ME. Are physicians asking about tobacco use and assisting with cessation? Results from the 2001-2004 national ambulatory medical care survey (NAMCS). Prev Med 2006; 43:472-6.

8. Thorndike AN, Regan S, Rigotti NA. The treatment of smoking by US physicians during ambulatory visits: 1994-2003. Am J Public Health 2007;97:187883.

9. Fiore MC, Jaén CR, Baker TB, et al. Treating tobacco use and dependence: 2008 update. Clinical practice guideline. Rockville, MD: U.S. Department of Health and Human Services; 2008:36.

10. Fiore MC. The new vital sign. Assessing and documenting smoking status. JAMA 1991;266:3183-4.

11. Boyle R, Solberg LI. Is making smoking status a vital sign sufficient to increase cessation support actions in clinical practice? Ann Fam Med 2004;2:22-5.

12. Maizlish NA, Ruland J, Rosinski ME, Hendry K. A systems-based intervention to promote smoking as a vital sign in patients served by community health centers. Am J Med Qual 2006;21:169-77.

13. Milch CE, Edmunson JM, Beshansky JR, Griffith JL, Selker HP. Smoking cessation in primary care: a clinical effectiveness trial of two simple interventions. Prev Med 2004;38:284-94.

14. Ahluwalia JS, Gibson CA, Kenney E, Wallace DD, Resnicow K. Smoking status as a vital sign. J Gen Intern Med 1999;14:402-8.

15. Rothemich SF, Woolf SH, Johnson RE, et al. Effect on cessation counseling of documenting smoking status as a routine vital sign: an ACORN study. Ann Family Med 2008;6:60-8.

16. Fiore MC, Bailey WC, Cohen SJ, et al. Treating tobacco use and dependence. Quick reference guide for clinicians. Rockville, MD: U.S. Department of Health and Human Services; 2000.

17. Thomson O'Brien MA, Freemantle N, Oxman AD, Wolf F, Davis DA, Herrin J. Continuing education meetings and workshops: effect on professional prac- 
tice and health care outcomes. Cochrane Database Syst Rev 2001;(2):CD003030.

18. Davis D, O'Brien MA, Freemantle N, Wolf F, Mazmanian P, Taylor-Vaisey A. Impact of formal continuing medical education: do conferences, workshops, rounds, and other traditional continuing education activities change physician behavior or health care outcomes? JAMA 1999;282:867-74.

19. Wilson A, McDonald P. Comparison of patient questionnaire, medical record, and audio tape in assessment of health promotion in general practice consultations. BMJ 1994;309:1483-5.

20. Nicholson JM, Hennrikus DJ, Lando HA, McCarty
MC, Vessey J. Patient recall versus physician documentation in report of smoking cessation counseling performed in an inpatient setting. Tob Control 2000;9:382-388.

21. Pbert L, Adams A, Quirk M, Hebert J, Ockene JK, Luippold RS. Patient exit interview as an assessment of physician-delivered smoking intervention: a validation study. Health Psychol 1999;18:183-8.

22. Conroy MB, Majchrzak NE, Silverman CB, et al. Measuring provider adherence to tobacco treatment guidelines: a comparison of electronic medical record review, patient survey, and provider survey. Nicotine Tob Res 2005;7(Supl 1):S35-S43. 